\title{
Estudo da implementação de discussões de atualidades e sua influência em um grupo PET
}

DOI: 10.37702/2175-957X.COBENGE.2021.3538

Hiago Fernandes Oliveira Pereira - hiago.petmec@gmail.com

Universidade Federal de Uberlândia

Rua Maria das Dores Dias 850

38408-206 - Uberlândia - MG

Larissa Silva Barbosa - larissasb25@outlook.com

Universidade Federal de Uberlândia

Rua Nelson de Oliveira 628

38408-204 - Uberlândia - MG

Pedro Henrique Maia Zandonadi - pedromaia.petmec@gmail.com

Universidade Federal de Uberlândia

Rua João Velasco de Andrade 218

38408-202 - Uberlândia - MG

Ana Marta Souza - anamartaengenharia@gmail.com

Universidade Federal de Uberlândia

Rua Jangadeiros 201

38412-016 - Uberlândia - MG

Resumo: Em um cenário de um mercado de trabalho competitivo, muitas vezes é esperado que os participantes do processo seletivo demonstrem domínio de certas habilidades pessoais, como capacidade de trabalhar em grupo, saber se expressar em um grupo de pessoas, saber lidar com opiniões diferentes, entre outras. Essas habilidades, denominadas soft skills, tornaram-se um ponto chave diferencial na graduação dos estudantes de engenharia. Portanto, os membros do Programa de Educação Tutorial da Faculdade de Engenharia Mecânica (PETMEC) da Universidade Federal de Uberlândia (UFU) buscaram contribuir para o aprimoramento destas habilidades com a realização de duas atividades, nomeadas como Papo PET e Conversação, as quais consistem na realização de debates alternados em português e um idioma de língua estrangeira. Sendo assim, no presente artigo visou-se estudar a influência e o impacto dessas atividades no desenvolvimento pessoal de membros atuais e de ex-membros do grupo PETMEC. 
Foi elaborado um formulário para os entrevistados questionando a opinião destes quanto à contribuição das referidas atividades em suas habilidades pessoais e à importância dada às soft skills no mercado de trabalho e na graduação. Analisando as 26 respostas ao formulário, pode-se concluir que as atividades em questão são de grande relevância para o desenvolvimento das soft skills dos membros do PETMEC, indo de encontro com a importância que estes atribuem às soft skills no mercado de trabalho. Além disso, o Papo PET e a Conversação são eventos aliados à uma formação mais ampla, contribuindo com uma capacitação além das habilidades puramente técnicas de engenharia.

Palavras-chave: soft skills, mercado de trabalho, aperfeiçoamento, debate, PET 


\section{ESTUDO DA IMPLEMENTAÇÃO DE DISCUSSÕES DE ATUALIDADES E SUA INFLUÊNCIA EM UM GRUPO PET}

\section{INTRODUÇÃO}

A aprendizagem e o desenvolvimento de habilidades interpessoais são de fundamental importância para um profissional. Algumas atividades realizadas durante a graduação ajudam no desenvolvimento das conhecidas soft skills, ou seja, contribuem para a comunicação, trabalho em equipe, proatividade, oratória e exposição de ideias. Para Agante (2005), são designadas de soft skills as competências não técnicas como a comunicação, criação de empatia, confiança com grupos e capacidade de resiliência em um ambiente de trabalho com incerteza. Essas habilidades são desenvolvidas através de atividades, cursos e experiências.

Quando se trata de engenharia, Nilsson (2010) diz que o programa educacional deveria focar menos em conteúdos essenciais para o currículo do engenheiro e priorizar as competências generalistas e habilidades transversais, incluindo competências interpessoais. Isso porque na engenharia o foco, na maioria das vezes, é no conhecimento técnico e no desenvolvimento físico e matemático presente nos componentes da área.

As habilidades ditas não técnicas tornaram-se um elemento primordial no que diz respeito às exigências dos empregadores nos dias atuais. Deste modo, os jovens estudantes de engenharia precisam estar cientes de tamanha necessidade a fim de adentrarem ao mundo corporativo (COSTA, 2015). Ou seja, esses fatores se mostram importantes na formação do engenheiro.

Além disso, os recém-formados em engenharia carecem de um treinamento adicional que thes proporcione maior embasamento com relação às soft skills capacidade de aprendizagem contínua, ética profissional e moral, capacidade em comunicar-se em inglês, bom desenvolvimento profissional, além de habilidades interpessoais e empreendedoras (COSTA, 2015). Perante o exposto, a comunicação em inglês e o aprendizado de uma segunda língua são imprescindíveis no mercado atual, então a conversação em inglês veio como uma atividade que estimula o aprendizado e o treino da língua, além de desafiar os alunos com temas diferenciados.

Diante disso, o grupo PETMEC desenvolveu duas atividades: o Papo PET e a conversação. A dinâmica aplicada durante o Papo PET visa auxiliar no desenvolvimento de exposição de ideias, do ouvir atentamente a opinião dos colegas de equipe, entender as diferentes abordagens do tema discutido e se posicionar. A conversação possui o mesmo objetivo, mas com um desafio maior: realizar a atividade na língua inglesa. Portanto, o principal objetivo deste trabalho é analisar a influência dessas atividades na formação dos alunos que participam do Programa de Educação Tutorial da Faculdade de Engenharia Mecânica (PETMEC) da Universidade Federal de Uberlândia (UFU), uma vez que um melhor entendimento do impacto das atividades em questão é um ponto importante para o aperfeiçoamento das dinâmicas e para a contribuição na graduação dos discentes. 


\section{METODOLOGIA}

\subsection{Aplicação no grupo}

Pautado em um de seus objetivos - elevar o desempenho acadêmico de seus membros e desenvolver habilidades pessoais em equipe - o grupo do Programa de Educação Tutorial PETMEC UFU implementa e estimula atividades que desenvolvam as anteriormente definidas soft skills em seus membros, que são alunos de graduação das engenharias Mecânica, Mecatrônica e Aeronáutica. Desde sua criação, em 1992, o grupo desenvolveu inúmeras atividades internas com membros da Faculdade de Engenharia Mecânica.

O Papo PET, implementado no grupo desde 2013, permite um ganho elevado na capacidade de argumentação e discussão, além de fomentar a curiosidade e interesse por temas atuais e de grande importância para um conhecimento de mundo, essencial para a formação pessoal. A atividade é realizada pelos membros do grupo durante as reuniões, intercalada com a Conversação em língua estrangeira semanalmente. Seu objetivo é a discussão de atualidades, podendo estas terem ou não relação com a área de engenharia e tecnologia, ampliando os conhecimentos dos membros do grupo através do debate acerca de um tema previamente definido. A cada edição, um integrante é responsável por escolher e expor o tema a ser discutido a todos os outros membros, sendo ele previamente comunicado e referenciado para que todos possam se informar e chegar ao debate e apresentação do tema com uma base para a discussão.

Em complemento a isso, o PETMEC historicamente preza pela proficiência de seus integrantes em língua estrangeira, o que se evidenciava nos relatórios anuais desde 2005 até a implementação da Conversação, visto que eram relatados os cursos de idiomas e níveis de proficiência de cada integrante. A partir de 2009, a Conversação em Língua Estrangeira foi instituída no grupo de maneira a aprimorar o conhecimento dos membros em outro idioma, principalmente o Inglês. Para isso, um ciclo de palestras e seminários em língua inglesa foi instituído mensalmente, com temas relacionados às pesquisas dos próprios integrantes e outros que levavam a discussões entre os membros.

Anos mais tarde, com o início da prática do Papo PET, a Conversação foi unida ao mesmo projeto, levando ao cenário atual em que as duas atividades ocorrem semanalmente e de maneira intercalada. A Conversação tem como principal objetivo elevar a capacidade de compreensão e fala dos membros em outro idioma, além de permitir um ambiente livre e sem julgamentos para que todos os níveis de proficiência possam se desenvolver. Desse modo, os temas expostos durante as Conversações se assemelham muito com aqueles do Papo PET, fazendo com que semanalmente haja uma discussão pertinente de um tema atual tanto em português como em língua estrangeira, contribuindo em um amplo espectro para o desenvolvimento pessoal dos membros.

Alguns exemplos de temas propostos e discutidos nas edições do Papo PET e Conversação em língua estrangeira são:

- Novos meios de mobilidade: Táxi x Aplicativos;

- Uso excessivo de tecnologias, seus ganhos e prejuízos;

- Temas associados à legalidade de práticas como aborto e uso de drogas;

- Temas relacionados a filmes e livros, além de acontecimentos históricos;

- Temas associados a políticas públicas e sociais como desarmamento, maioridade penal, impunidade no Brasil, casamento homoafetivo;

- Temas gerais da atualidade como uso de energias renováveis, depressão e ansiedade no ambiente universitário, consumo de carne e veganismo. 


\subsection{Coleta de dados}

Foi realizada uma pesquisa através de um formulário online na plataforma Google Formulários. O público-alvo foram os alunos que já fizeram ou fazem parte do PET da Engenharia Mecânica. O link foi enviado individualmente para cada aluno. As respostas obtidas foram armazenadas no próprio formulário e em uma planilha, usando o programa Excel. Ao fim da pesquisa, foram obtidas 26 respostas.

Com base nos dados obtidos no questionário, foram levantados os resultados e as conclusões sobre o impacto das atividades na formação do discente, além de verificar a importância das soft skills. Os resultados foram analisados através da porcentagem de cada opção dada, sendo possível analisar qualitativamente cada pergunta e obter uma conclusão.

\section{RESULTADOS E INFLUÊNCIA}

O formulário aplicado conta com três seções, sendo a primeira destinada à caracterização dos entrevistados, informando nome, curso de graduação e tempo que esteve ligado ao grupo PETMEC e participando dos Papo PET e Conversações. A segunda seção coletou dados quanto à opinião dos entrevistados sobre as contribuições das atividades em questão no desenvolvimento de suas habilidades pessoais. Por fim, a terceira e última seção do formulário exigiu dos entrevistados que estes atribuíssem notas de 1 a 5 para a importância das soft skills e o desenvolvimento destas. Os resultados serão apresentados por seção.

\subsection{Seção 1 - Quanto aos entrevistados}

Como o preenchimento do formulário contou com a colaboração de membros atuais e ex-membros do PETMEC, foi questionado primeiro quanto à distribuição dos entrevistados entre os três cursos de graduação ofertados pela Faculdade de Engenharia Mecânica (FEMEC) da UFU. O resultado pode ser observado na Figura 1 abaixo.

Figura 1 - Gráfico da distribuição dos entrevistados entre os cursos de graduação da FEMEC.

Curso:

26 respostas

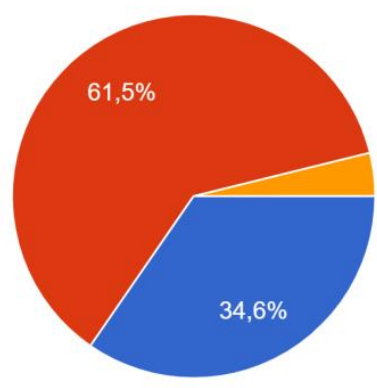

Engenharia Aeronáutica

Engenharia Mecânica

Engenharia Mecatrônica

Fonte: Formulário elaborado pelos autores. 
Pode-se observar então que dentre os 26 participantes, $61,5 \%$ destes, ou seja, 16 pessoas, cursam atualmente ou cursaram Engenharia Mecânica, enquanto 34,6\%, correspondente a 9 pessoas, cursam ou cursaram Engenharia Aeronáutica. Quando se trata da Engenharia Mecatrônica, somente 1 pessoa assinalou essa opção e este fato está indo de encontro à baixa participação dos alunos da Engenharia Mecatrônica nos processos seletivos do grupo PETMEC.

Ademais, caracterizando os entrevistados quanto ao tempo em que estiveram ligados ao Programa de Educação Tutorial, pode-se ver no gráfico apresentado na Figura 2 que metade destes estiveram no grupo PETMEC pelo período de 1 a 2 anos. Além disso, 30,8\%, correspondente a 8 pessoas, estiveram presentes por mais de 2 anos e uma menor parte, correspondente a 19,2\%, ou 5 pessoas, participaram por menos de 1 ano.

Figura 2 - Gráfico da distribuição dos entrevistados por tempo de participação.

Por quanto tempo você foi membro do PET, participando dos PapoPET e Conversações?

26 respostas

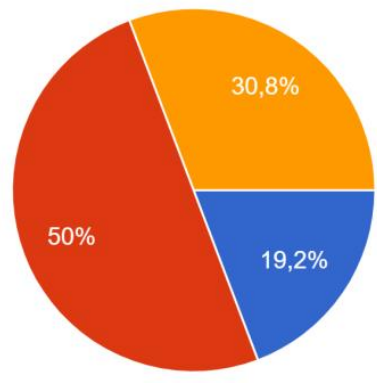

Fonte: Formulário elaborado pelos autores.

\subsection{Seção 2 - Quanto a contribuição dos PapoPET e Conversações}

Nessa parte do formulário, foram apresentadas aos entrevistados algumas afirmações sobre os Papo PET e Conversações e cinco opções de respostas, sendo elas: concordo totalmente, concordo parcialmente, neutro, discordo parcialmente e discordo totalmente.

Na Figura 3 abaixo nota-se que a maioria das respostas converge para a conclusão de que os Papo PET e as Conversações contribuíram positivamente para uma melhoria na maneira de se comunicar, uma vez que $84,6 \%$ dos participantes concordaram totalmente ou parcialmente com a afirmação e somente 1 participante, correspondente à $3,8 \%$, disse que discorda parcialmente. Nessa pergunta, 3 pessoas optaram pela opção "neutro". 
Figura 3 - Gráfico da distribuição das respostas dos entrevistados para a 1ª afirmação da seção 2.

"Os PapoPET e Conversações contribuíram para melhorar a maneira como eu me comunico"

26 respostas
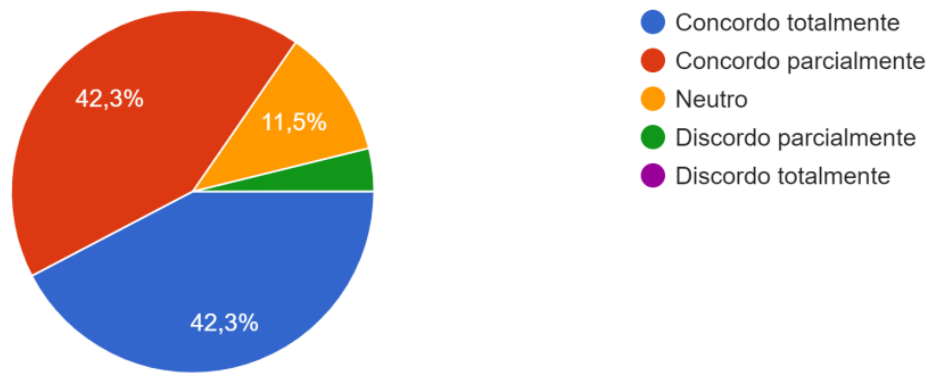

Fonte: Formulário elaborado pelos autores.

Agora, quanto à contribuição das atividades Papo PET e Conversação na melhoria do senso crítico dos participantes, as respostas estão na Figura 4. Mais da metade $(57,7 \%)$ dos entrevistados apontaram que concordam totalmente, que somados aos que concordam parcialmente, alcança-se aproximadamente $85 \%$ de concordância com a afirmação apresentada. O restante corresponde a 3 respostas na opção "neutro" e novamente apenas uma discordando, no caso assinalando a opção "discordo totalmente". Acredita-se que a alta porcentagem de concordância possa estar atrelada ao fato de frequentemente serem feitas abordagens de temas polêmicos e sociais nos debates.

Figura 4 - Gráfico da distribuição das respostas dos entrevistados para a 2ª afirmação da seção 2.

"Os PapoPET e Conversações contribuiram para melhorar meu senso crítico"

26 respostas

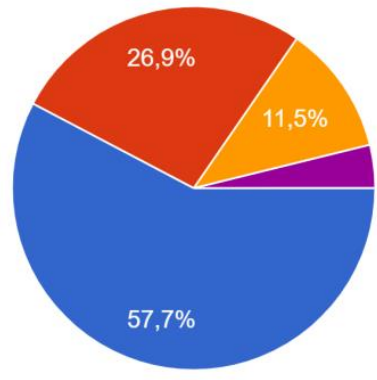

Concordo totalmente

Concordo parcialmente

Neutro

Discordo parcialmente

Discordo totalmente

Fonte: Formulário elaborado pelos autores.

Quando se pediu para avaliar a contribuição dos Papo PET e Conversações na capacidade de lidar com divergências de opiniões, não foi obtida nenhuma resposta em discordância à afirmação apresentada. Nessa pergunta, 15,4\%, equivalente a 4 respostas, foram na opção "neutro" e as demais foram igualmente divididas entre as duas categorias de "concordo". As respostas estão abaixo na Figura 5. Uma vez que nos debates é muito comum haver discordâncias de opiniões, os resultados mostram que a 
maioria dos participantes aprenderam a lidar com essas situações mais facilmente devido aos Papo PET e às Conversações.

Figura 5 - Gráfico da distribuição das respostas dos entrevistados para a 3ª afirmação da seção 2.

"Os PapoPET e Conversações contribuíram para melhorar minha capacidade de lidar com divergências de opiniões"

26 respostas

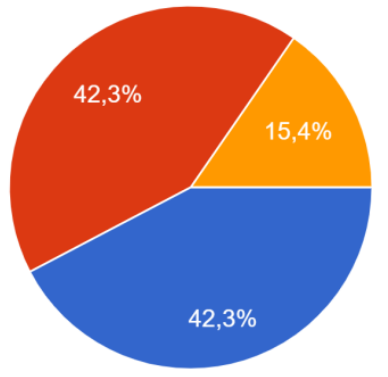

Concordo totalmente

Concordo parcialmente

Neutro

Discordo parcialmente

Discordo totalmente

Fonte: Formulário elaborado pelos autores.

Uma outra afirmação passada aos entrevistados é quanto à contribuição das atividades em questão na capacidade de expressar um posicionamento para um grupo de pessoas. Pela primeira vez, observa-se na Figura 6 que a porcentagem de "concordo parcialmente" superou a de "concordo totalmente", sendo $50 \%$ e $34,5 \%$, respectivamente. Os 15,4\% restantes foram na opção "neutro". Uma possível justificativa, analisando essas respostas, é que essa habilidade de expressar posicionamentos é muito cobrada em todas as atividades do PET, uma vez que o grupo sempre cobra sugestões dos membros. Portanto, esperava-se que os entrevistados realmente notassem melhorias nessa competência, ainda mais quando exercida nos Papo PET e Conversações, mesmo que não sejam essas as atividades em que os membros mais trabalharam essa habilidade.

Figura 6 - Gráfico da distribuição das respostas dos entrevistados para a 4ª afirmação da seção 2.

"Os PapoPET e Conversações contribuiram para melhorar minha capacidade de expressar um posicionamento para um grupo de pessoas"

26 respostas

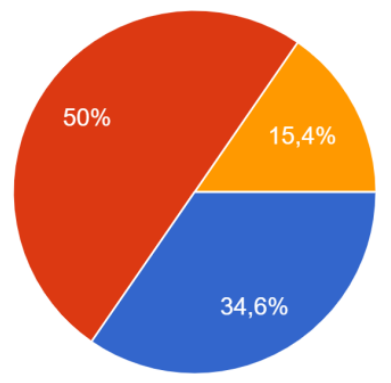

Concordo totalmente

Concordo parcialmente

Neutro

Discordo parcialmente

Discordo totalmente

Fonte: Formulário elaborado pelos autores. 
Por fim, a última afirmação repassada na seção 2 do formulário foi sobre a influência das Conversações na capacidade de expressar-se em outro idioma. Essa afirmação, dentre todas as outras, foi a que teve maior percentual de discordância e menor percentual de concordância. 7,7\% das respostas assinalaram "discordo totalmente" e 3,8\% assinalaram "discordo parcialmente", totalizando $11,5 \%$ de discordância. Por outro lado, mesmo sendo o menor percentual de concordância de todas, ainda sim é maioria das respostas com $69,3 \%$, sendo $46,2 \%$ do "concordo totalmente" e $23,1 \%$ do "concordo parcialmente". As demais respostas foram na opção "neutro". O gráfico está apresentado abaixo na Figura 7.

Figura 7 - Gráfico da distribuição das respostas dos entrevistados para a 5ª afirmação da seção 2.

"Os PapoPET e Conversações contribuiram para melhorar minha capacidade de me expressar em outro idioma"

26 respostas

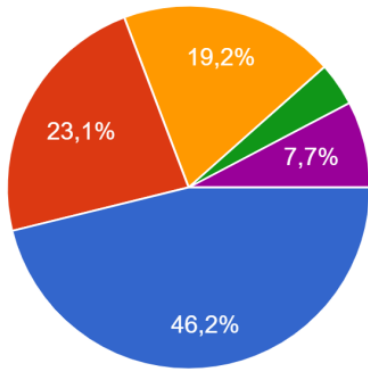

Concordo totalmente

Concordo parcialmente

Neutro

Discordo parcialmente

Discordo totalmente

Fonte: Formulário elaborado pelos autores.

Apresentados esses dados, cabe questioná-los. Nesse caso, essa distribuição de respostas pode ser atribuída ao fato de que muitos membros e ex-membros já entram e participam do grupo PETMEC com um alto nível de proficiência na língua estrangeira e, portanto, não se nota grandes melhorias. Outra observação é que durante as Conversações a participação e o engajamento são menores do que nos Papo PET, então muitos membros do grupo simplesmente não exercem com frequência a língua estrangeira, não usufruindo integralmente dessa oportunidade. Portanto, como o tempo da atividade é limitado e nem todos se sentem confortáveis em participar, acabam sendo mais beneficiados aqueles que participam ativamente dos debates.

\subsection{Seção 3 - Quanto às soft skills}

Nessa terceira e última seção do formulário, foi pedido aos entrevistados que respondessem ao que foi pedido levando em consideração o seguinte conceito apresentado na divisão do formulário: "são designadas de soft skills as competências não técnicas como a comunicação, criação de empatia, confiança com grupos e capacidade de resiliência em um ambiente de trabalho com incerteza (Agante, 2005)".

Na primeira questão pediu-se para que fosse classificado de 1 a 5 a importância que 0 entrevistado atribui às soft skills analisando o cenário do mercado de trabalho. Nesse caso, 1 corresponde a "nada importante" e 5 a "muito importante". A distribuição das respostas pode ser observada no gráfico abaixo, na Figura 8. Cabe ressaltar que a 
média obtida é de 4,88, ou seja, os membros e ex-membros do grupo PETMEC classificam como muito importante as soft skills no mercado de trabalho.

Figura 8 - Gráfico da distribuição das respostas dos entrevistados para a 1ª questão da seção 3.

Analisando o cenário do mercado de trabalho, classifique a importância que você atribui às soft skills:

26 respostas

30

20

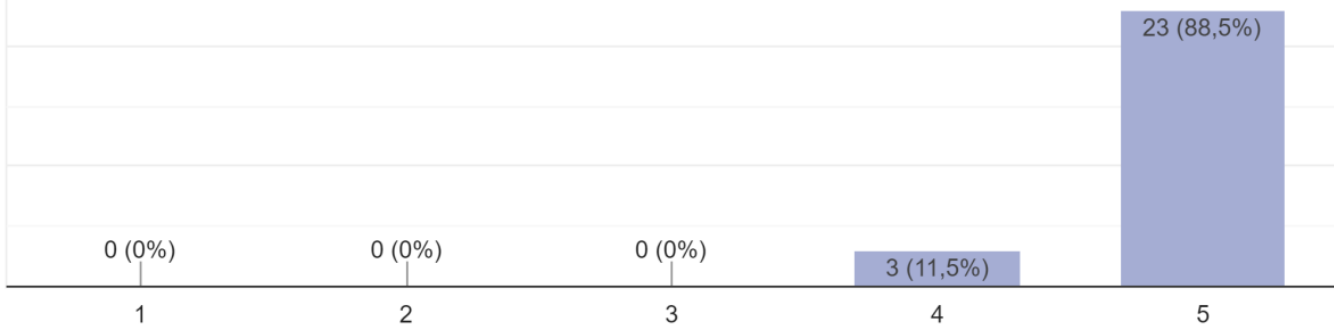

Fonte: Formulário elaborado pelos autores.

Já a segunda questão relaciona diretamente os Papos PET e as Conversações com as soft skills, uma vez que solicita aos participantes que classifiquem a contribuição destas atividades no desenvolvimento das soft skills. Nesse caso, 1 é o equivalente a "não contribuiu" e 5 o equivalente a "contribuiu bastante". As respostas obtidas estão na Figura 9 em formato de gráfico. Com esse questionamento aos entrevistados, obteve-se a média igual a 4, com uma distribuição mais diversificada das respostas, mas com somente uma resposta atribuindo nota 2 e nenhuma atribuindo nota 1.

Figura 9 - Gráfico da distribuição das respostas dos entrevistados para a 2ª questão da seção 3.

Classifique a contribuição dos PapoPET e Conversações no desenvolvimento de suas soft skills: 26 respostas

15

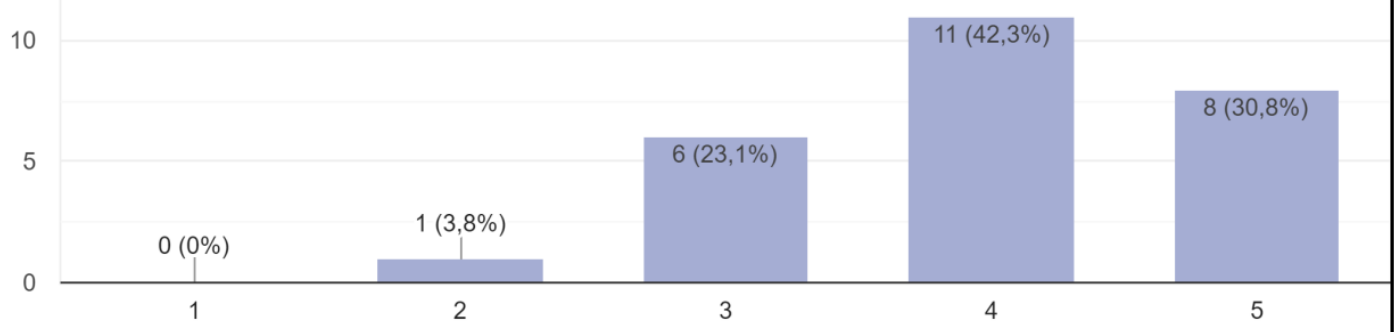

Fonte: Formulário elaborado pelos autores. 
Concluindo o formulário, a última e terceira questão da seção 3 também solicitados entrevistados uma classificação de 1 a 5 , mas dessa vez quanto a importância que é dada às soft skills ao longo da graduação ao se analisar a realidade dos estudantes de engenharia. Para essa questão, 1 é atribuído a "não é dada importância" e 5 a "é dada muita importância". Nesse caso, a média das respostas é 2,73 o que leva ao entendimento que, na visão dos membros e ex-membros do Programa de Educação Tutorial, é dada pouca importância às soft skills durante a graduação. O gráfico encontrase a seguir, na Figura 10.

Figura 10 - Gráfico da distribuição das respostas dos entrevistados para a 3ª questão da seção 3.

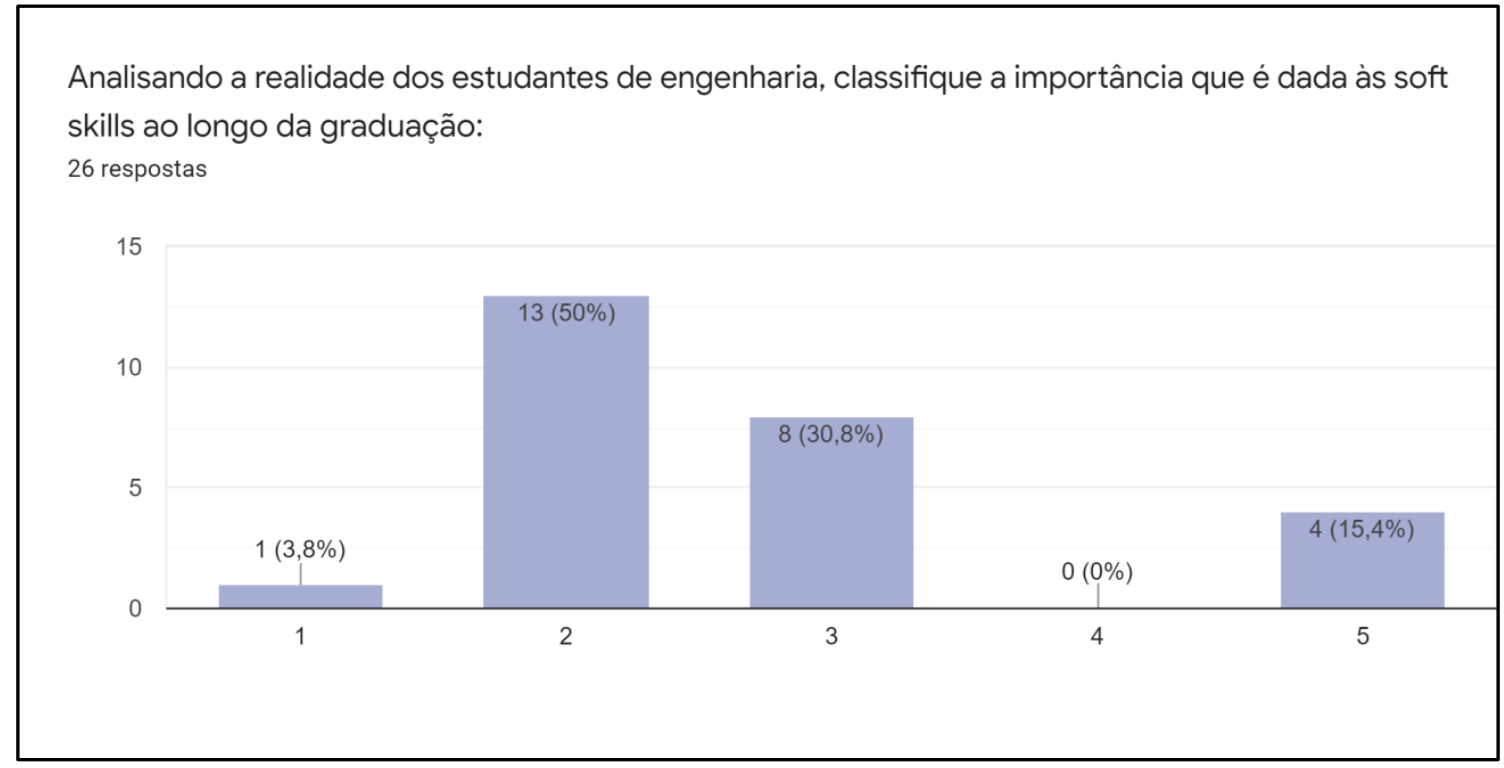

Fonte: Formulário elaborado pelos autores.

Logo, com os dados dessa seção já apresentados, é importante apontar possíveis interpretações. Como foi atribuída média 4 para a contribuição dos debates no desenvolvimento das soft skills dos participantes, pode ser interpretado que as atividades analisadas no trabalho vão de encontro com a importância dessas skills no mercado de trabalho, a qual obteve média 4,88 nas respostas do formulário. Além disso, como foi atribuída média 2,73 para a importância dada às soft skills ao longo da graduação, então as atividades Papo PET e Conversação são entendidas como complementares à graduação, oferecendo paralelamente uma oportunidade de aprimoramento das habilidades dos membros do grupo PETMEC em detrimento à pouca importância que é dada a essas habilidades durante a graduação.

\section{CONSIDERAÇÕES FINAIS}

É notório que, muitas vezes, os alunos ficam focados em suas áreas de atuação e preocupações acadêmicas na universidade, o que acaba contribuindo para uma negligência em relação aos acontecimentos externos a ela. Tal situação é não apenas prejudicial para o indivíduo, como também para a sociedade como um todo em que ele se insere. Em tempos de grandes extremismos e dualidades, faz-se ainda mais necessário que os cidadãos sejam capazes de discutir acerca de temas atuais e evoluam suas capacidades argumentativas e de respeito às opiniões alheias. Já na esfera profissional, o mercado de trabalho procura profissionais versáteis, que sejam capazes de expor e 
defender um ponto de vista da melhor maneira e que saibam ouvir opiniões e críticas para melhorarem.

Ademais, o mundo globalizado de hoje requer da sociedade uma grande capacidade comunicativa, que envolve o conhecimento de novos idiomas. Além de permitir acesso a culturas distintas e benefícios pessoais, o conhecimento de línguas estrangeiras tornou-se requisito para diversos postos de trabalho mesmo dentro do Brasil. Com isso, o desenvolvimento de habilidades não apenas comunicativas, mas também argumentativas em outro idioma vem se tornando fator essencial para a vida profissional, e não apenas um diferencial como outrora se via.

Os resultados do presente estudo vão de encontro à importância dada a esse assunto pelo mercado de trabalho. Foi possível comprovar que as atividades realizadas dentro do grupo contribuem para o desenvolvimento de habilidades interpessoais, e possuem, certamente, relevância para a formação mais completa do engenheiro. Portanto, as atividades realizadas com os membros do grupo PETMEC são pertinentes e complementam a graduação, ajudando a melhorar as denominadas soft skills, mesmo que elas não tenham a devida atenção dentro do meio acadêmico da engenharia.

\section{Agradecimentos}

Ao PETMEC (Programa de Educação Tutorial da Faculdade de Engenharia Mecânica) da Universidade Federal de Uberlândia pelo apoio para a realização do presente trabalho.

\section{REFERÊNCIAS}

AGANTE, L. A importância das soft skills na vida profissional, Dinheiro Vivo.

Disponível em: https://www.dinheirovivo.pt/carreiras/a-importancia-das-soft-skills-na-vidaprofissional/. Acesso em: 15 abr. 2021.

COSTA, N. A importância das competências transversais (soft skills) na formação do engenheiro. 2015. 82 p. Monografia - Escola de Engenharia de Lorena. Universidade de São Paulo, Lorena, 2015. Disponível em: https://sistemas.eel.usp.br/bibliotecas/monog rafias/2015/MIQ15031.pdf. Acesso em: 13 abr. 2021.

NILSSON, Staffan. Enhancing individual employability: the perspective of engineering graduates. Education + Training. 2010, Vol. 52, 6/7.

PETMEC UFU. Relatório Anual das Atividades PETMEC - Ano base 2005. Disponível em: http://www.pet.mecanica.ufu.br/o-que-é-o-pet. Acesso em: 21 abr. 2021.

PETMEC UFU. Relatório Anual das Atividades PETMEC - Ano base 2009. Disponível em: http://www.pet.mecanica.ufu.br/o-que-é-o-pet. Acesso em: 21 abr. 2021.

PETMEC UFU. Relatório Anual das Atividades PETMEC - Ano base 2013. Disponível em: http://www.pet.mecanica.ufu.br/o-que-é-o-pet. Acesso em: 21 abr. 2021.

PETMEC UFU. Relatório Anual das Atividades PETMEC - Ano base 2019. Disponível em: http://www.pet.mecanica.ufu.br/o-que-é-o-pet. Acesso em: 21 abr. 2021.

PETMEC UFU. Relatório Anual das Atividades PETMEC - Ano base 2020. Disponível em: http://www.pet.mecanica.ufu.br/o-que-é-o-pet. Acesso em: 21 abr. 2021. 


\title{
PAPO PET AND CONVERSAÇÃO: AN IMPLEMENTATION STUDY OF ACTUALITIES DISCUSSION AND ITS INFLUENCE IN A PET GROUP
}

\begin{abstract}
Considering a competitive job market scenario, its often expected that the candidates of a job offer demonstrate competence of certain personal abilities such as group work, self-expression in a group, manage different point of views, among many others. Those abilities are called soft skills and became a key aspect to undergraduate engineering students. Therefore, the members of the Tutorial Education Program of the Mechanical Engineering Department (PETMEC) from the Federal University of Uberlândia (UFU) looked for improving these skills with Papo PET and Conversação activities, which consists of alternate debates in Portuguese and a foreign language. Hence, the present article aimed to study the influence and the impact of the Papo PET and Conversações on the personal development of PETMEC'scurrent and ex members. A form was created to gather responses from the target public about their opinions regarding the influence of these activities on the improvement of personal skills and the relevance of soft skills on the job market and their undergraduate degree. After the analysis of 26 obtained answers, it is understood that the referred activities are of great relevance for the soft skills development of PETMEC members, in accordance with the significance of these skills for the professional life.
\end{abstract}

Keywords: soft skills, job market, improvement, debate, PET. 